\title{
Electronically Controllable Phase Shifter with Progressive Impedance Transformation at K Band
}

\author{
Mohamed T. ElKhorassani ${ }^{1}$, Angel Palomares-Caballero ${ }^{1, * \mathbb{D}}$, Antonio Alex-Amor ${ }^{1,2}$, \\ Cleofás Segura-Gómez ${ }^{1}$, Pablo Escobedo ${ }^{3}{ }^{\mathbb{D}}$, Juan F. Valenzuela-Valdés ${ }^{1}$ and Pablo Padilla ${ }^{1} \mathbb{C}$ \\ 1 Department of Signal Theory, Telematics and Communications, Universidad de Granada-CITIC, \\ 18071 Granada, Spain; taha.elkhorasssani@gmail.com (M.T.E.); aalex@gr.ssr.upm.es (A.A.-A.); \\ cleofas@correo.ugr.es (C.S.-G.); juanvalenzuela@ugr.es (J.F.V.-V.); pablopadilla@ugr.es (P.P.) \\ 2 Information Processing and Telecommunications Center, Universidad Politécnica de Madrid, \\ 28040 Madrid, Spain \\ 3 Bendable Electronics and Sensing Technologies (BEST) Group, School of Engineering, University of Glasgow, \\ Glasgow G12 8QQ, UK; pablo.escobedo@glasgow.ac.uk \\ * Correspondence: angelpc@ugr.es
}

Received: 11 November 2019; Accepted: 29 November 2019; Published: 1 December 2019

\begin{abstract}
This communication presents the design of a two-port electronically tunable phase shifter at $\mathrm{K}$ band. The phase shifter consists of a $3 \mathrm{~dB}$ hybrid coupler loaded with reflective phase-controllable circuits. The reflective circuits are formed by varactors and non-sequential impedance transformers which increase the operational bandwidth and the provided phase shift. The final phase shifter design is formed by two loaded-coupler stages of phase shifting to guarantee a complete phase turn. An $18 \mathrm{GHz}$ phase shifter design with dynamic range of 600 degrees of phase shift is depicted in this document. The prototype is manufactured and validated through measurements showing good agreement with the simulation results.
\end{abstract}

Keywords: microwave tunable phase shifters; $3 \mathrm{~dB} / 90^{\circ}$ coupler; $\mathrm{K}$ band

\section{Introduction}

Antenna arrays have been one of the most used structural strategies for the design of directive antennas in microwave ranges with demanding radiation requirements [1]. In the last years, planar substrate-based antennas have been present in many phased array designs due to the low manufacturing costs, easy integration, and low weight and profile [2,3]. This is particularly of interest in array structures such as reflectarrays and transmitarrays. In the case of the reflectarrays, the conformed reflectors are replaced by planar array structures that introduce at each array cell the desired phase shift, whose value is the one provided by the corresponding point of the equivalent reflector [4]. The transmitarrays are based on the same working principle, but the structure replaced by the periodic array is a lens instead of a reflector [5]. Both options have become a real alternative in reception/transmission systems whose specifications imply demanding requirements in terms of matching, directivity, gain, radiation pattern, or aperture efficiency [6]. The phase shifting strategy for both reflectarray- and transmitarray-based antennas depends on the system architecture and is typically obtained by a phase-delay circuit at each radiating cell of the array $[7,8]$. This phase shift can be fixed in design, yielding a passive device, or tunable, yielding an active antenna. The tunable nature of the phase shifter can be provided either mechanically or electronically [6,9]. Many of the designs of electronically controllable phase shifters are based on reflective inductance-capacitance (LC) tunable circuits [10], most of them focused on frequencies up to $3 \mathrm{GHz}$ [11-14]. This latter approach is the one that guides this work, leading to the design of electronically reconfigurable phase shifters at higher microwave ranges [10]. In this paper 
we present a K band electronically tunable phase shifter based on reflective circuits with progressive impedance transformers and varactors. These impedance transformers provide an enhanced frequency bandwidth and an increase in the phase variation produced in the phase shifter [15].

The relevance of our work is based on the combination of three main aspects, which are: the use of impedance transformers for increasing the phase variation range, the unitary phase shifter cascading, and the high operating frequency $(18 \mathrm{GHz})$. These three elements combined confer interest to our work and provide a significant contribution related to previous works, such as [10] and [15]. In particular, the idea of using of impedance transformers proposed in [15] is now validated through prototypes. The document is organized as follows: Section 2 depicts the phase shifter working principle and presents the shifter design, while Section 3 presents the validation through the manufacture and measurement of a prototype. Finally, conclusions are outlined in Section 4.

\section{Materials and Methods: Phase Shifter Working Principle and Design}

The working principle of the device is based on the combination of two microwave elements: a $3 \mathrm{~dB} / 90$ hybrid coupler and a couple of reflective tunable circuits. The reflective circuits are connected to the $-3 \mathrm{~dB} / 90^{\circ}$ and $-3 \mathrm{~dB} / 180^{\circ}$ output ports (ports 3 and 4 , respectively), while the isolated port of the $3 \mathrm{~dB} / 90^{\circ}$ coupler becomes the output port (port 2). Figure 1 shows the connection scheme. The input signal (port 1) is conducted through the coupler to ports 3 and 4 with a phase difference of $90^{\circ}$ between them. The signal reflections in ports 3 and 4 steer both signals again towards the coupler, adding the reflective circuit tunable phase variation $(\Delta \varphi)$. As a result, port 1 receives no signal (opposite phase summation of identical signals) and all the available power is routed to port 2 with a phase shift of $\Delta \varphi$. For the reflective circuits, we propose the use of a progressive impedance transformation, for enlarging the achievable phase range.

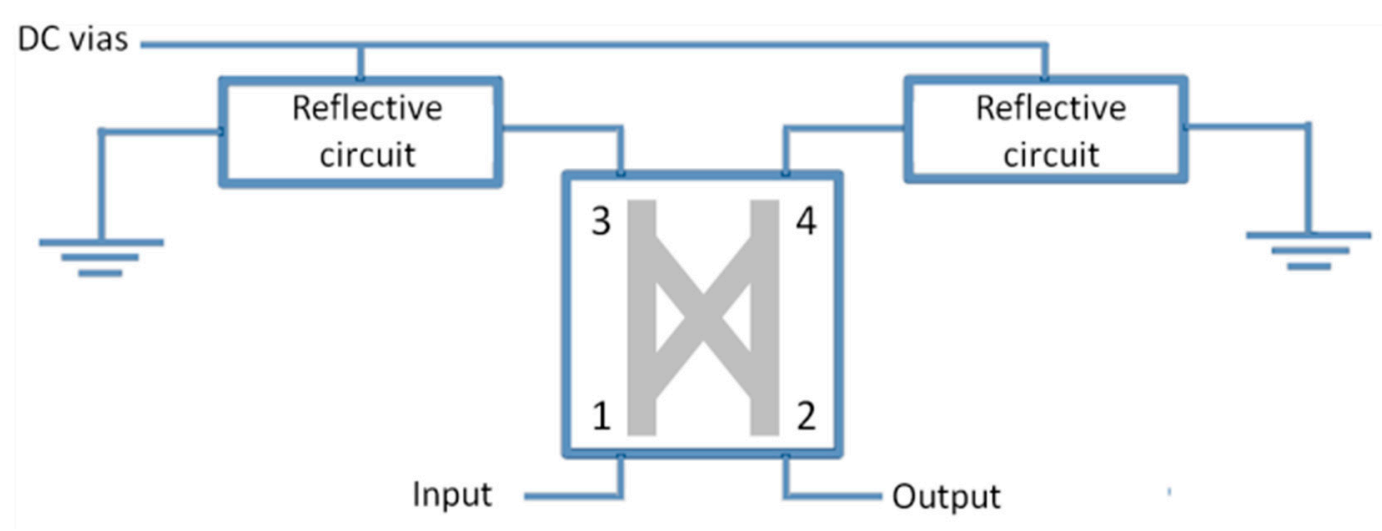

Figure 1. Scheme of the reflection-based phase shifter.

\subsection{Baseline $3 \mathrm{~dB} / 90^{\circ}$ Coupler}

The basic element of the phase shifter is a conventional $3 \mathrm{~dB} / 90^{\circ}$ coupler. The coupler is designed for a working frequency of $18 \mathrm{GHz}$ with a bandwidth higher than $2 \mathrm{GHz}$ considering $-20 \mathrm{~dB}$ in the $\left|\mathrm{S}_{11}\right|$ level. The coupler symmetry imposes identical amplitude values levels at ports 3 and 4 , with an accurate $90^{\circ}$ phase difference between these ports. Figure 2 provides the model of the microstrip coupler, along with its performance results. 


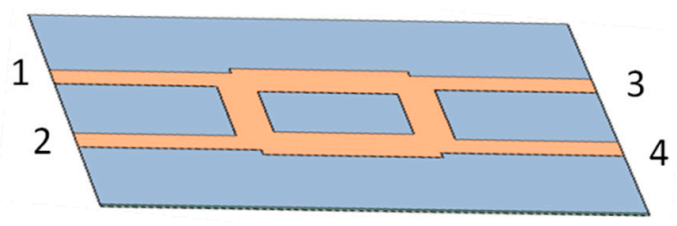

(a)

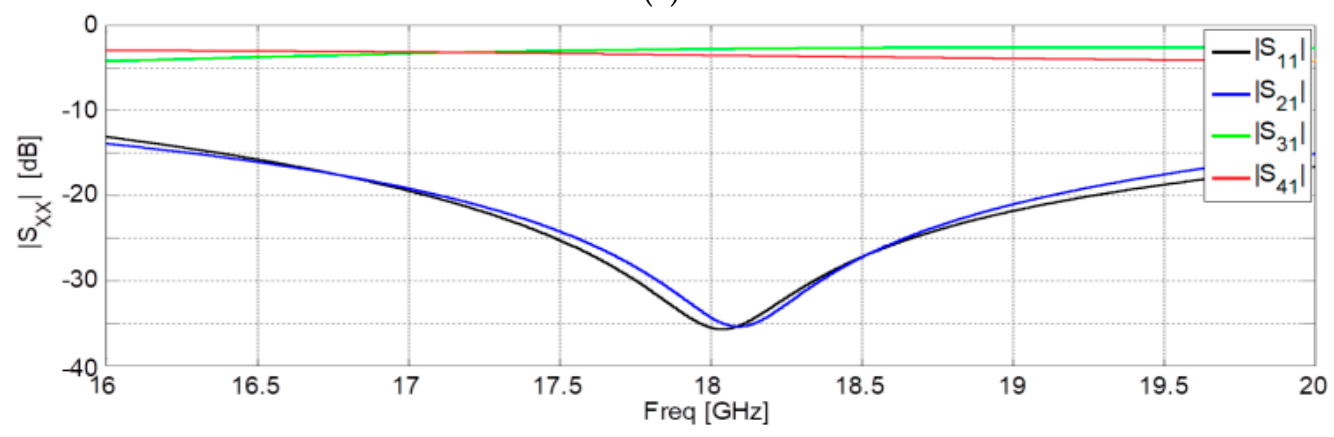

(b)

Figure 2. Three decibel hybrid coupler: (a) microstrip design; (b) design performance ( $\left|S_{X X}\right|$ values).

\subsection{Reflective LC Circuit with Progressive Impedance Transformation}

The key element of the phase shifter that provides its reconfigurability is a reflective circuit. It is composed of a LC circuit with a short-circuit to the ground plane at the end. The capacitive effect is provided by a varactor while the inductive effect is provided by narrowing a section in the microstrip line (high impedance section). The varactor used for this design is the MA46585 varactor, with a capacity range of $0.13 \mathrm{pF}$ to $2.2 \mathrm{pF}$. The connection to the ground plane is made with a metallic via. Due to the low resistive value of the varactors $(\sim 9 \Omega)$, it is necessary to add a progressive impedance transformation to the reflective circuit, in order to improve the performance of the reflective element. For the 9-to-50 $\Omega$ transformation an impedance conversion based on two cascaded quarter-wavelength transformers is designed. The sequential transformation of the impedance in two steps impedance (i.e., transformation of $50 \Omega$ to $25 \Omega$ and $25 \Omega$ to $9 \Omega$ ) requires higher microstrip widths. These widths are impracticable in a size-reduced device. The alternative is a non-sequential transformation in two steps: to avoid low line impedances, a new relation between impedances can be set up, according to Equation (1). This principle of impedance transformation is illustrated in Figure 3, where $Z_{x}$ are the characteristic impedances of the microstrip sections.

$$
Z_{\text {in }}=\frac{Z_{0}^{2}}{Z_{x}}=\frac{Z_{0}^{2}}{Z_{1}^{2} / Z_{0}}=\frac{Z_{0}^{3}}{Z_{1}^{2}}
$$

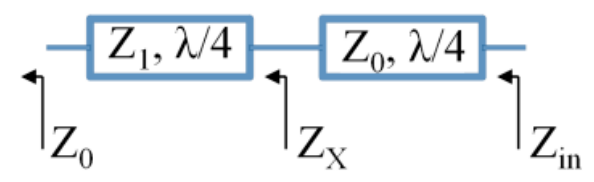

Figure 3. Non-sequential cascaded $\lambda / 4$ impedance transformers according to Equation (1).

The non-sequential transformation requires a quarter-wavelength section of impedance $Z_{1}$ of $118 \Omega$, which means a thinner microstrip section. Therefore, two cascaded quarter-wave impedance transformers with characteristic impedance of $118 \Omega$ and $50 \Omega$, respectively, are used to transform the circuit impedance from $50 \Omega$ to $9 \Omega$ along a width bandwidth. Figure 4 provides the circuit model of the non sequential transformer, and its design outcomes. 


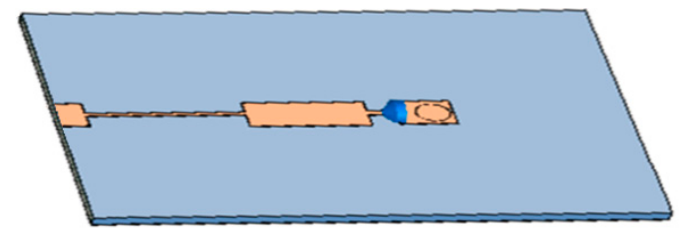

(a)

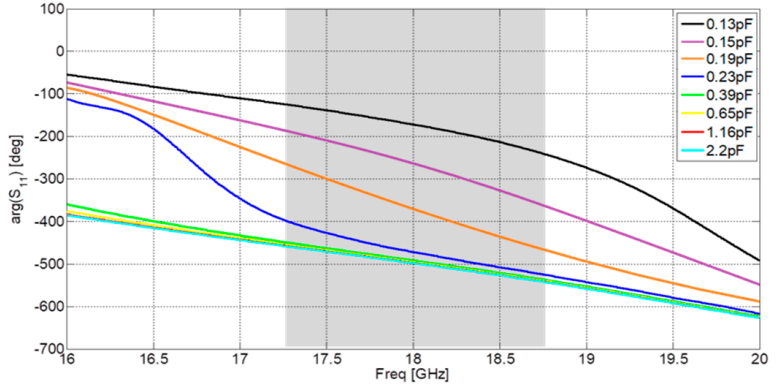

(b)

Figure 4. Design results of the reflective LC circuit with progressive impedance transformation: (a) simulation design; (b) phase shift produced.

The total phase variation is of around $290^{\circ}$ for the complete capacity range [0.13-2.2 $\mathrm{pF}$, but with a non-linear behavior. However, this non-linearity is compensated by the DC-voltage/capacity relation. Compared to the case without progressive impedance transformation, the latter provides a phase shift of around $220^{\circ}$. This means an improvement of at least $70^{\circ}$ due to the progressive impedance transformation, for the same capacity range.

\subsection{Complete Phase Shifter Design and Performance}

The phase shift provided by a loaded coupler is not enough to guarantee a phase variation higher than $360^{\circ}$. Therefore, in the complete device, two cascaded sets are connected. Figure 5 provides the complete phase shifter model, together with its performance results. The desired band in the design is at least of $1.5 \mathrm{GHz}$, centered at $18 \mathrm{GHz}$.

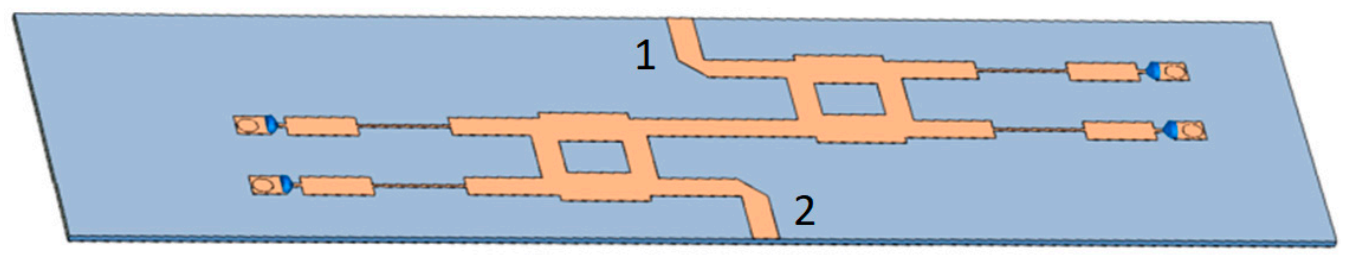

(a)

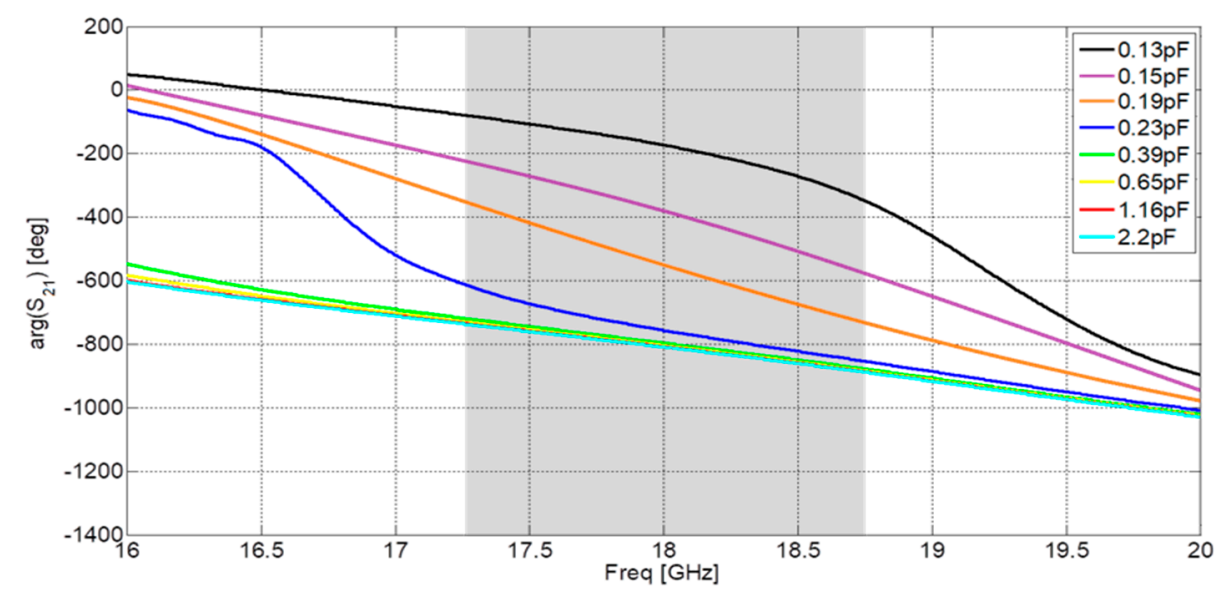

(b)

Figure 5. Cont. 


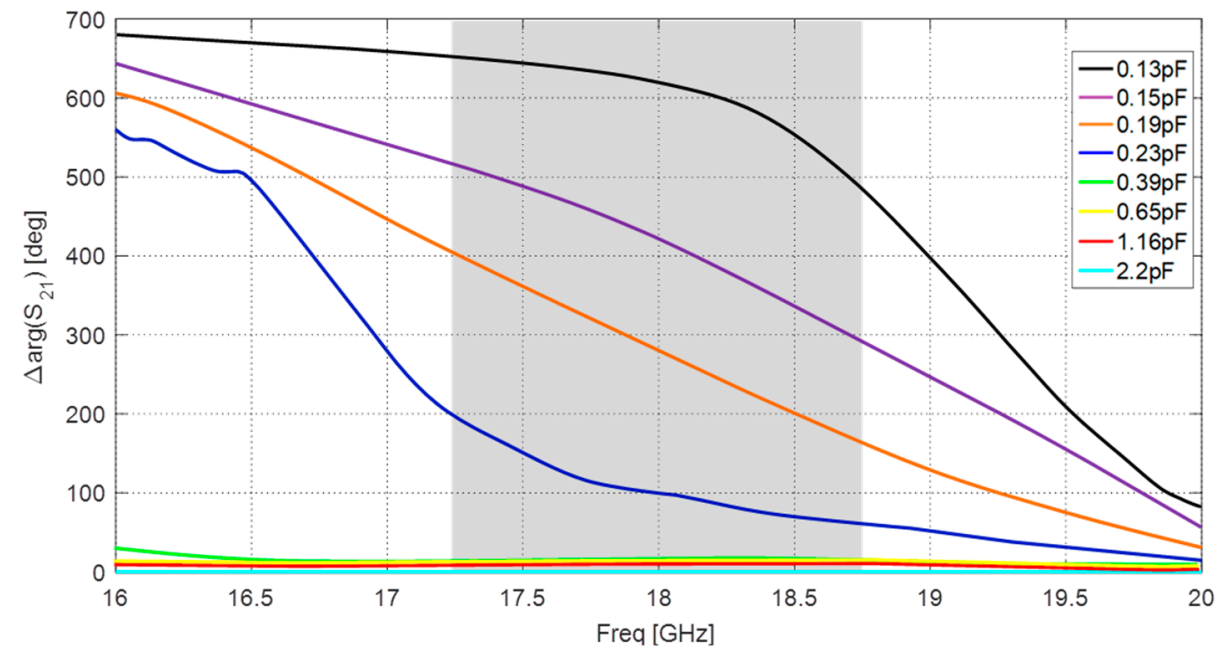

(c)

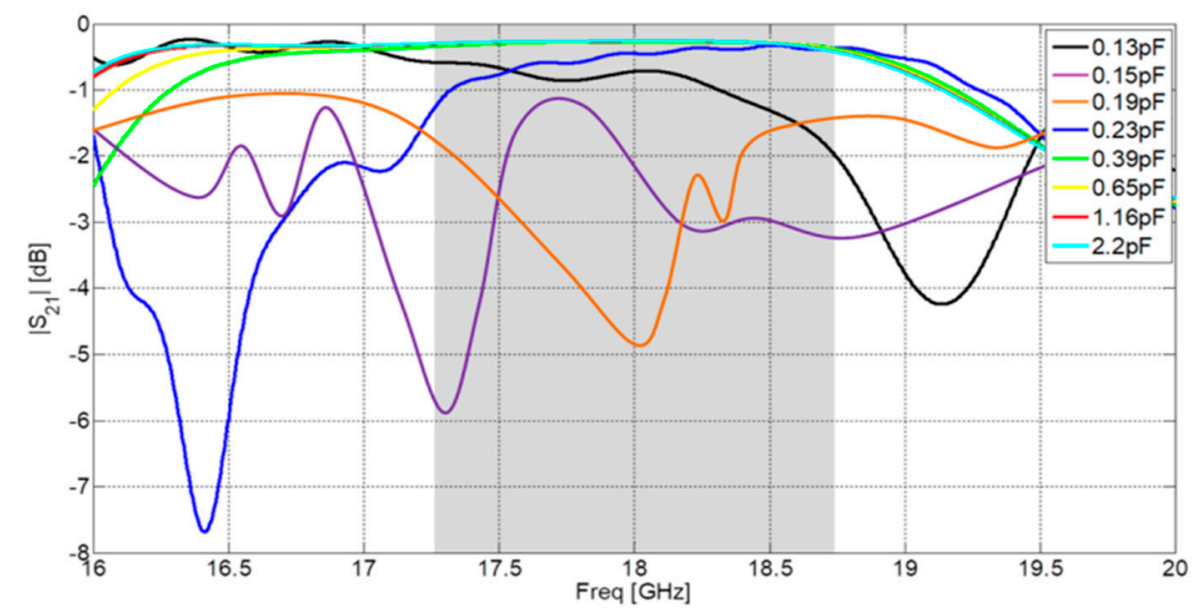

(d)

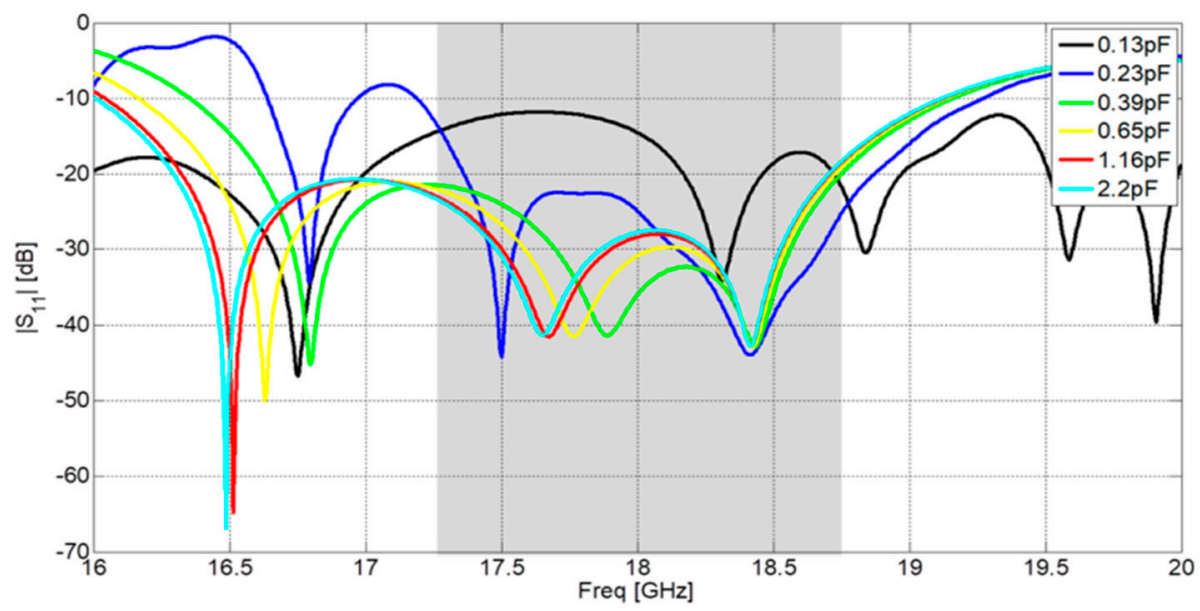

(e)

Figure 5. Simulation results of the $18 \mathrm{GHz}$ phase shifter, (a) design; (b) $\arg \left(\mathrm{S}_{21}\right)$; (c) $\Delta \arg \left(\mathrm{S}_{21}\right)$ (related to $\min \arg \left(\mathrm{S}_{21}\right)$ value, $\left.2.2 \mathrm{pF}\right) ;(\mathbf{d})\left|\mathrm{S}_{21}\right| ;(\mathbf{e})\left|\mathrm{S}_{11}\right|$. 
The shifter design presents proper matching in the desired band and a loss level of around $6 \mathrm{~dB}$ in the worst case. Considering that the working band is centered at $18 \mathrm{GHz}$, this loss level can be considered an appropriate value, if compared to commercially available devices. The phase shifting capability of the shifter is around $600^{\circ}$, almost twice the phase variation obtained for the reflective circuit, as expected.

The high operating frequency band (central frequency $18 \mathrm{GHz}$ ) is highly demanding and limiting in terms of design and manufacturing. In our work, best efforts are concentrated in having an easy-manufacturing device with reasonable performance results. Thus, the device is based on planar microstrip technology, which is almost in its frequency limit of operation. All the manufacturing and mounting has been carried out in our laboratory. The photolithography manufacturing process introduces a manufacturing size uncertainty of around 100 microns. The substrate is a Neltec N9217 ( $0.508 \mathrm{~mm}$ thickness, relative permittivity of 2.17 , and $<0.0008$ loss tangent). The lumped elements (the MA46585 varactors) are welded by means of conductive epoxy. The device circuit design dimensions are provided in Table 1.

Table 1. Device circuit design dimensions (mm).

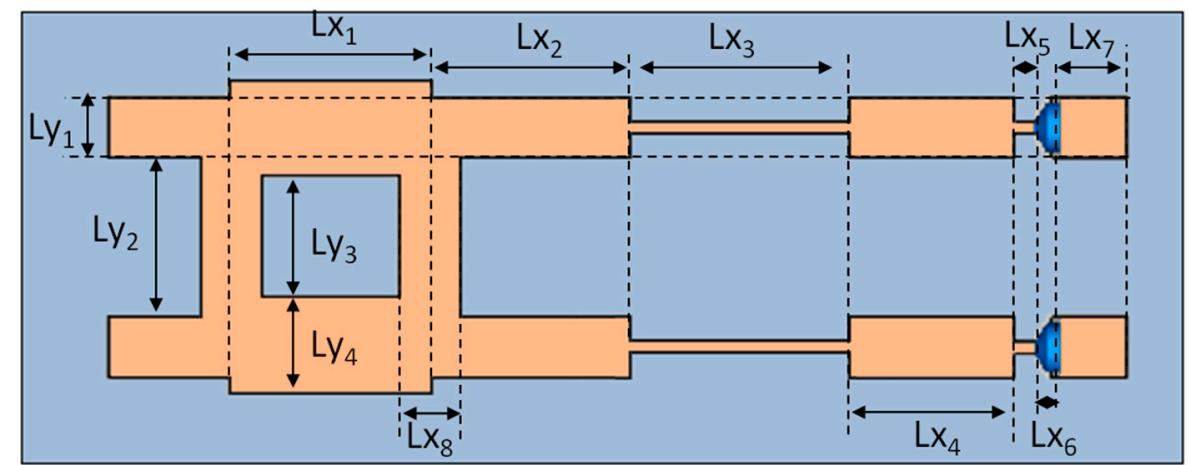

\begin{tabular}{cccccccccccc}
\hline $\mathbf{L}_{\mathbf{x} 1}$ & $\mathbf{L}_{\mathbf{x} \mathbf{2}}$ & $\mathbf{L}_{\mathbf{x} \mathbf{3}}$ & $\mathbf{L}_{\mathbf{x} \mathbf{4}}$ & $\mathbf{L}_{\mathbf{x} \mathbf{5}}$ & $\mathbf{L}_{\mathbf{x} \mathbf{6}}$ & $\mathbf{L}_{\mathbf{x} 7}$ & $\mathbf{L}_{\mathbf{x} 8}$ & $\mathbf{L}_{\mathbf{y} \mathbf{1}}$ & $\mathbf{L}_{\mathbf{y} \mathbf{2}}$ & $\mathbf{L}_{\mathbf{y} \mathbf{3}}$ & $\mathbf{L}_{\mathbf{y} \mathbf{4}}$ \\
\hline 3.43 & 3.06 & 3.49 & 2.5 & 0.47 & 0.3 & 1 & 0.75 & 0.75 & 2.25 & 1.75 & 1.25 \\
\hline
\end{tabular}

\section{Results: Phase Shifter Prototype Performance}

The design has been validated through a prototype. Figure 6.a yields some details of the shifter prototype, and the TRL (transmission-reflection-line) kit for the calibration prior to the measuring process. The measurement results are provided in the rest of the figures in Figure 6, in order to validate its proper functioning and performance.
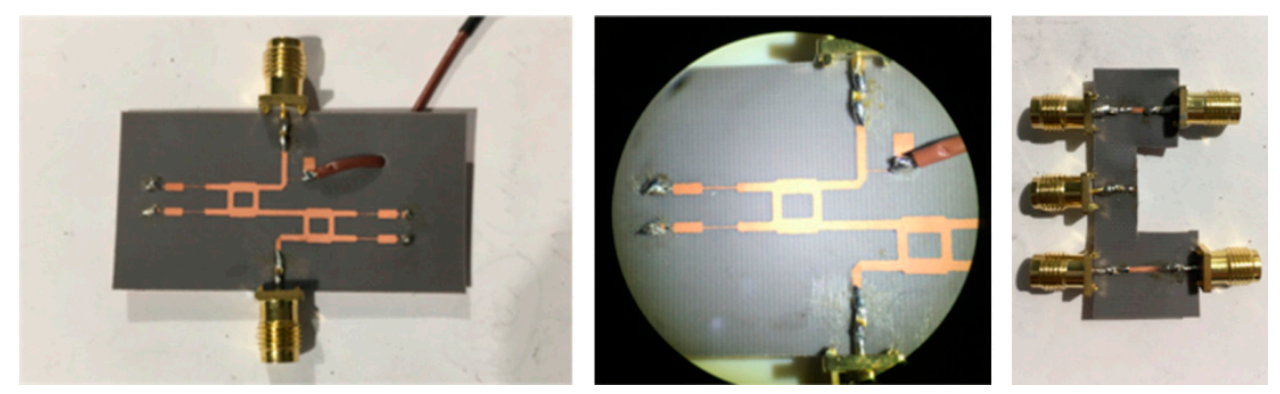

(a)

Figure 6. Cont. 
Figure 6. Cont.

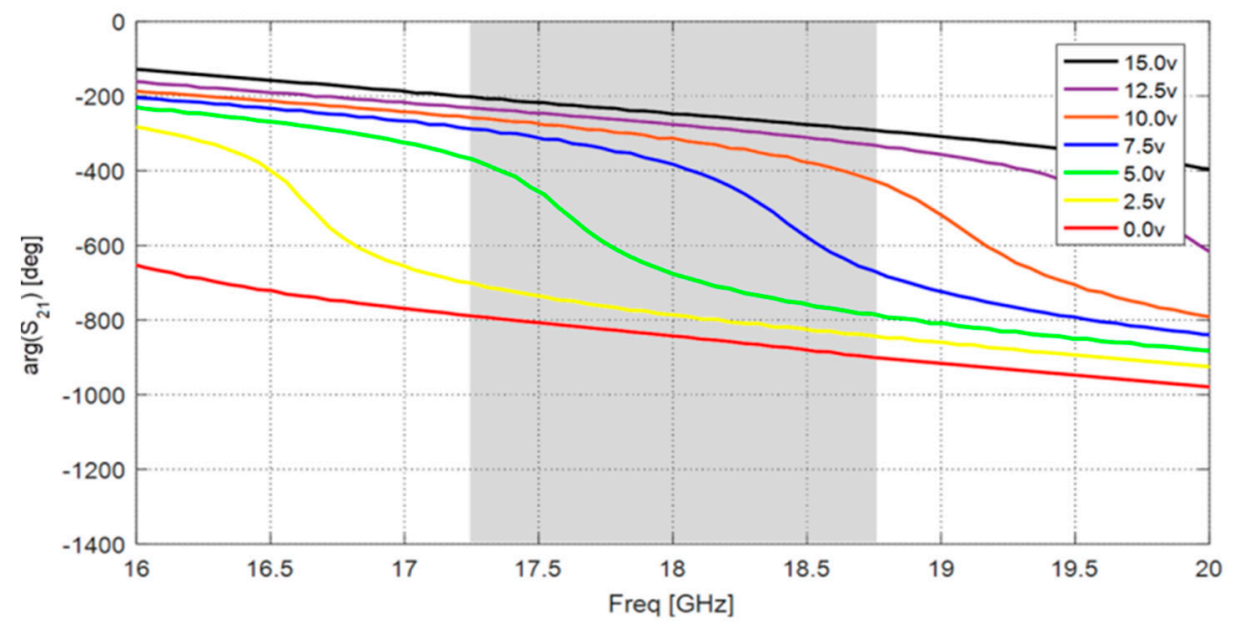

(b)

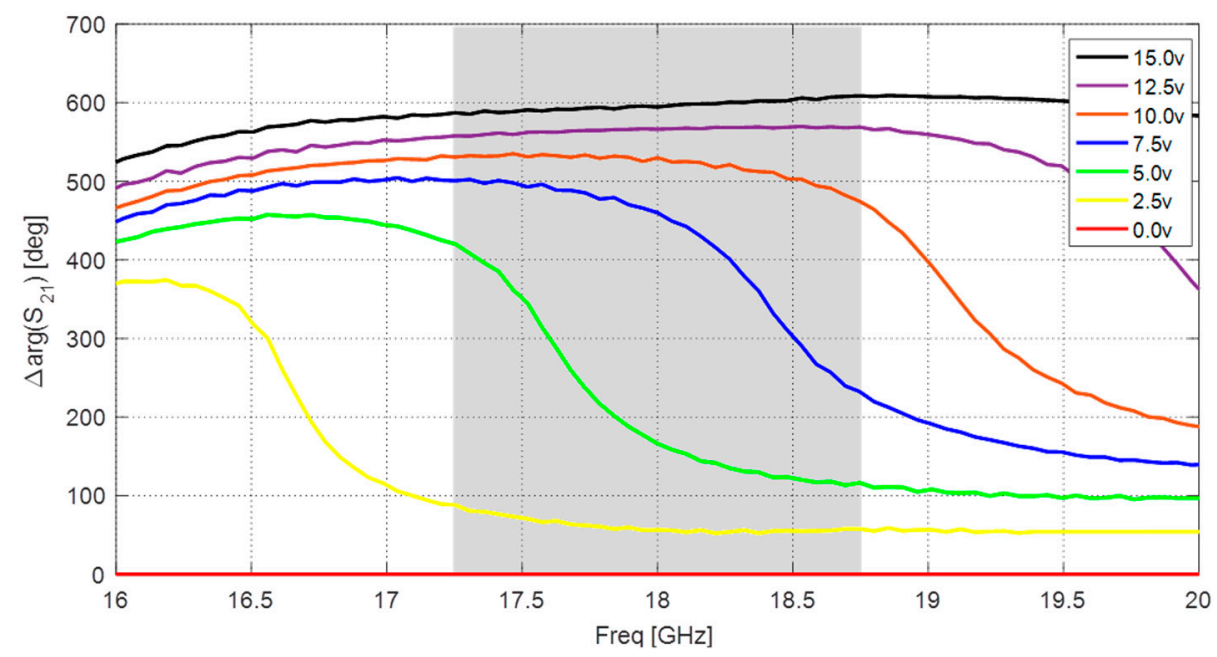

(c)

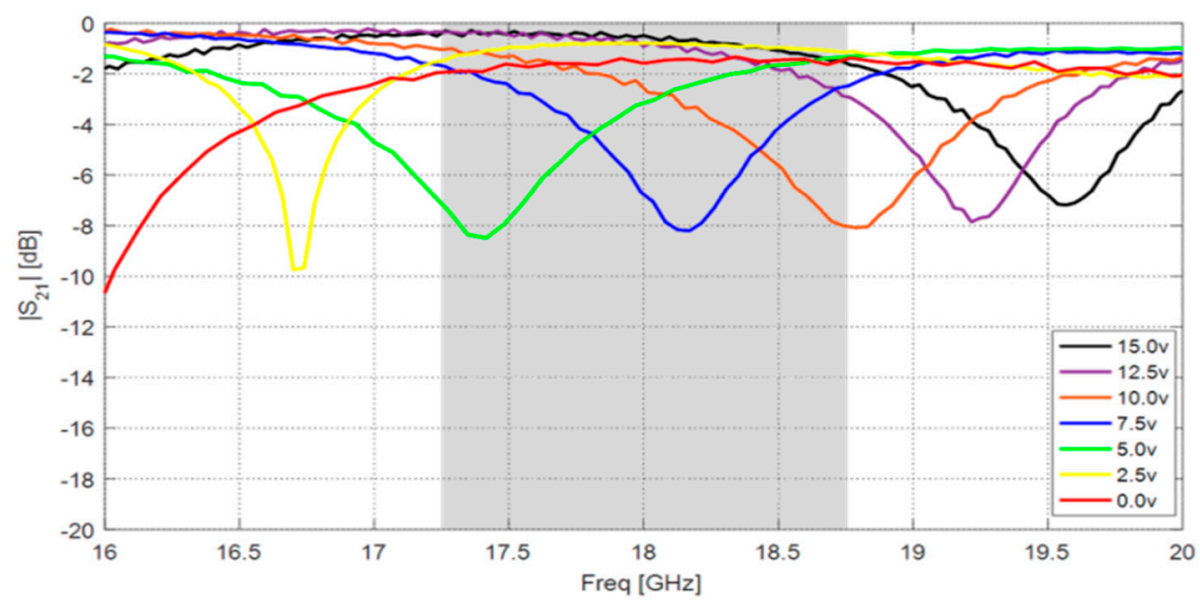

(d) 


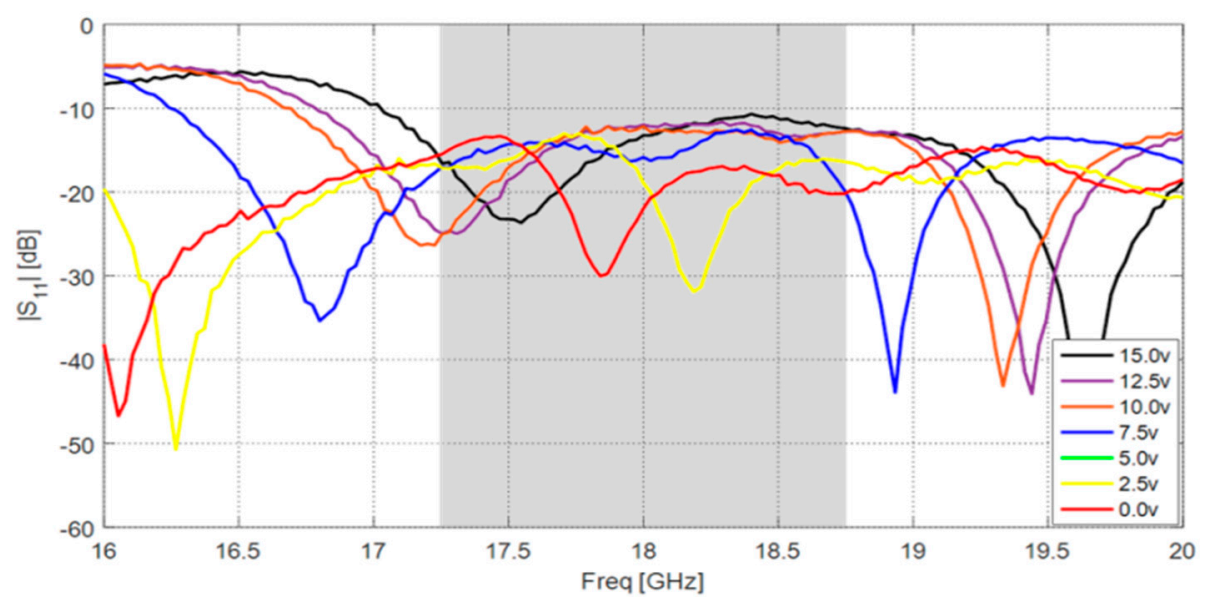

(e)

Figure 6. Prototype results of the $18 \mathrm{GHz}$ phase shifter: (a) prototype and TRL kit (central image corresponds to a microscope zoom view); (b) $\arg \left(\mathrm{S}_{21}\right) ;(\mathbf{c}) \Delta \arg \left(\mathrm{S}_{21}\right)$ (related to $\min \arg \left(\mathrm{S}_{21}\right)$ value, $\left.0 \mathrm{v}\right)$; (d) $\left|S_{21}\right| ;\left(\right.$ e) $\left|S_{11}\right|$.

As it can be noticed, there is a good agreement between the design performance and the prototype measurements. The insertion loss levels of the prototype are higher than the expected ones in the design. There exists a variety of possible causes for that: parasitic components of the varactor, conductive epoxy resistance, and roughness of the shifter printed line limits. For the sake of completeness, Figure 7 compares the simulation phase results with the experimental ones (Figure 7a), and provides an insight of the linearity of the device in terms of phase/voltage relation (Figure $7 \mathrm{~b}$ ).

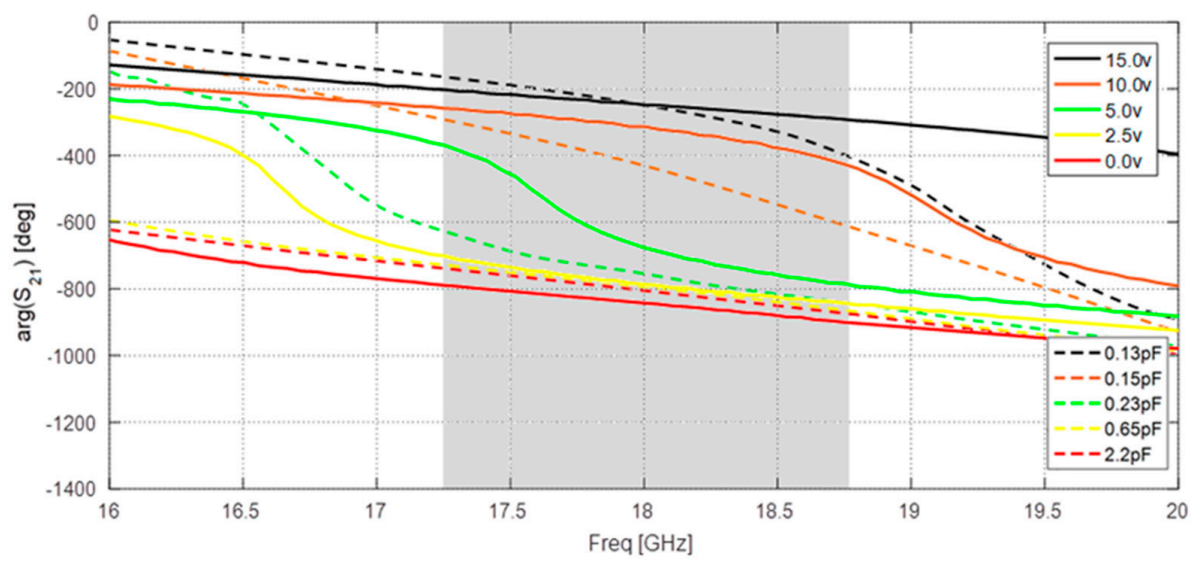

(a)

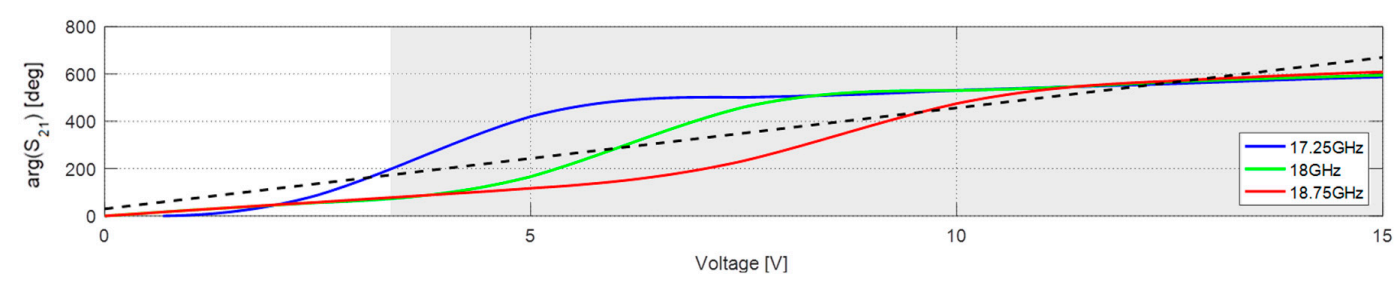

(b)

Figure 7. Phase shifting performance: (a) comparison between simulation and measurement phase results of the $18 \mathrm{GHz}$ phase shifter; (b) phase/voltage relation (the dashed line is the linear reference and the grey zone is the one that provides at least $360^{\circ}$ variation). 
Notice that the simulation results in Figure $7 \mathrm{a}$ are in terms of capacitance and the experimental ones are in terms of voltage. It must be noticed that there is a non-negligible difference between pairs of simulation and experimental curves. However, the phase range of variation is preserved and the phase shifter prototype is fully functional, only needing a voltage/phase conversion table. In addition, it must be pointed out that, although the phase/capacitance relation is not linear, it is compensated by additional non-linear behavior of the capacitance/voltage relation. It is worth mentioning that, although the device provides a quasi linear phase/voltage response at the central frequency, this linearity is strongly degraded at the band limits, as it can be seen in Figure $7 \mathrm{~b}$. However, since the prototype provides a complete phase shift of around $600^{\circ}$, part of this possible variation can be sacrificed in order to increase the linearity of the device, as detailed in Figure $7 \mathrm{~b}$, where the grey region is the one that provides at least $360^{\circ}$ phase variation.

For the sake of completeness, the next table (Table 2) provides a comparison of the performance of our device with the previously referenced literature works [10-15].

Table 2. Performance comparison of our device with the previously referenced literature works.

\begin{tabular}{ccccc}
\hline Work & Central Frequency & Bandwidth & Insertion Losses & Phase Shifting Range \\
\hline$[11]$ & $2 \mathrm{GHz}$ & $200 \mathrm{MHz}$ & $<4.6 \mathrm{~dB}$ & $240^{\circ}$ \\
{$[12]$} & $2 \mathrm{GHz}$ & $200 \mathrm{MHz}$ & $<1.6 \mathrm{~dB}$ & $385^{\circ}$ \\
{$[13]$} & $2.5 \mathrm{GHz}$ & $500 \mathrm{MHz}$ & $<1.3 \mathrm{~dB}$ & $150^{\circ}$ \\
{$[14]$} & $2.5 \mathrm{GHz}$ & $500 \mathrm{MHz}$ & $<1.2 \mathrm{~dB}$ & $130^{\circ}$ \\
{$[15]$} & $12 \mathrm{GHz}$ & $>1 \mathrm{GHz}$ & $<3.2 \mathrm{~dB}$ & $290^{\circ}$ \\
{$[10]$} & $12.5 \mathrm{GHz}$ & $>2 \mathrm{GHz}$ & $<3 \mathrm{~dB}$ & $460^{\circ}$ \\
This work & $18 \mathrm{GHz}$ & $>1.5 \mathrm{GHz}$ & $<8 \mathrm{~dB}$ & $600^{\circ}$ \\
\hline
\end{tabular}

\section{Conclusions}

This communication presents the design and validation through prototyping of a microstrip phase shifter at $18 \mathrm{GHz}$ for $\mathrm{K}$ band applications, with electronically controllable phase shift. The design is based on the use of loaded $3 \mathrm{~dB} / 90^{\circ}$ couplers. The performance of the reflective loads is improved due to the use of non-sequential impedance transformers. This design is particularly useful for reconfigurable phased arrays such as transmitarrays or reflectarrays, being fully integrable in such designs because of the reduced footprint. Furthermore, the shifter architecture is suitable for miniaturization, increasing the permittivity of the substrate. The dynamic phase range of the phase shifter is higher than $600^{\circ}$ for the entire shifter bandwidth $(>1.5 \mathrm{GHz})$. This phase range is higher than a complete phase turn of $360^{\circ}$, which allows the introduction of phase wrapping. The device losses are lower than $8 \mathrm{~dB}$.

Author Contributions: M.T.E., A.P.-C., and A.A.-A. designed and simulated the phase shifter. P.E., C.S.-G., and P.P. manufactured and measured the prototype. A.P.-C. and P.P. wrote the document. J.F.V.-V. and P.P. supervised the whole study. All the authors participated in revising the article.

Funding: This work has been partially supported by the TIN2016-75097-P, RTI2018-102002-A-I00, and EQC2018004988-P projects of the Spanish National Program of Research, Development, and Innovation and project B-TIC-402-UGR18 of Junta de Andalucía.

Conflicts of Interest: The authors declare no conflict of interest.

\section{References}

1. Bhattacharyya, A.K. Phased Array Antennas: Floquet Analysis, Synthesis, BFNs and Active Array Systems, 1st ed.; Wiley-Interscience: Newark, NJ, USA, 2006.

2. Bhartia, P.; Bahl, I.; Garg, R.; Ittipiboon, A. Microstrip Antenna Design Handbook; Artech House Publishers: Norwood, MA, USA, 2000.

3. Lee, K.; Tong, K. Microstrip Patch Antennas-Basic Characteristics and Some Recent Advances. Proc. IEEE 2012, 100, 2169-2180. 
4. Carrasco, E.; Barba, M.; Encinar, J.A. Aperture-coupled reflectarray element with wide range of phase delay. Electron. Lett. 2006, 42, 667-668. [CrossRef]

5. Padilla, P.; Muñoz-Acevedo, A.; Sierra-Castañer, M.; Sierra-Pérez, M. Electronically reconfigurable transmitarray at $\mathrm{Ku}$ band for microwave applications. IEEE Trans. Antennas Propag. 2010, 58, 2571-2579. [CrossRef]

6. Hum, S.V.; Perruisseau-Carrier, J. Reconfigurable Reflectarrays and Array Lenses for Dynamic Antenna Beam Control: A Review. IEEE Trans. Antennas Propag. 2014, 62, 183-198. [CrossRef]

7. Lau, J.Y.; Hum, S.V. A Planar Reconfigurable Aperture With Lens and Reflectarray Modes of Operation. IEEE Trans. Microw. Theory Tech. 2010, 58, 3547-3555. [CrossRef]

8. Padilla, J.L.; Padilla, P.; Valenzuela-Valdés, J.F.; Fernández, J.M. High-frequency radiating element and modified $3 \mathrm{~dB} / 90^{\circ}$ electronic shifting circuit with circular polarization for broadband reflectarray device cells. Electron. Lett. 2014, 50, 1042-1043. [CrossRef]

9. Silva, J.S.; Lima, E.B.; Costa, J.R.; Fernandes, C.A.; Mosig, J.R. Tx-Rx Lens-Based Satellite-on-the-Move Ka-Band Antenna. IEEE Antennas Wirel. Propag. Lett. 2015, 14, 1408-1411. [CrossRef]

10. Padilla, P.; Valenzuela-Valdés, J.F.; Padilla, J.L.; Fernández-González, J.M.; Sierra-Castañer, M. Electronically Reconfigurable Reflective Phase Shifter for Circularly Polarized Reflectarray Systems. IEEE Microw. Wirel. Compon. Lett. 2016, 26, 705-707. [CrossRef]

11. Lin, C.; Chang, S.; Chang, C.; Shu, Y. Design of a Reflection-Type Phase Shifter With Wide Relative Phase Shift and Constant Insertion Loss. IEEE Trans. Microw. Theory Tech. 2007, 55, 1862-1868. [CrossRef]

12. Burdin, F.; Iskandar, Z.; Podevin, F.; Ferrari, P. Design of Compact Reflection-Type Phase Shifters With High Figure-of-Merit. IEEE Trans. Microw. Theory Tech. 2015, 63, 1883-1893. [CrossRef]

13. An, B.; Chaudhary, G.; Jeong, Y. Wideband tunable phase shifter with low in-band phase deviation error using coupled line. IEEE Microw. Wirel. Compon. Lett. 2018, 28, 678-680. [CrossRef]

14. Chaudhary, G.; An, B.; Jeong, Y. In-band phase minimization method for wideband tunable phase shifter. Microw. Opt. Technol. Lett. 2019, 61, 537-541. [CrossRef]

15. ElKhorassani, M.T.; Vaquero, M.A.; Palomares, A.; Valenzuela-Valdés, J.F.; Padilla, P.; Touhami, N.A. Electronically tunable phase shifter with enhanced phase behaviour at $\mathrm{Ku}$ Band. In Proceedings of the 12th European Conference on Antennas and Propagation (EuCAP 2018), London, UK, 9-13 April 2018. 